\title{
Direct comparison of line edge roughness measurements by SEM and a metrological tilting-atomic force microscopy for reference metrology
}

\author{
Ryosuke Kizu $\odot$,* Ichiko Misumi $\odot$, Akiko Hirai $\odot$, and Satoshi Gonda $\odot$ \\ National Metrology Institute of Japan, National Institute of Advanced Industrial Science and \\ Technology, Tsukuba, Ibaraki, Japan
}

\begin{abstract}
Background: Conventional scanning electron microscopy (SEM) that is used for 2D top-view metrology, a classical line edge roughness (LER) measurement technique, is incapable of measuring 3D structures of a nanoscale line pattern. For LER measurements, SEM measurement generates a single line-edge profile for the 3D sidewall roughness, although the line-edge profile differs at each height in the 3D sidewall.

Aim: To develop an evaluation method of SEM-based LER measurement techniques and to verify how the 3D sidewall shape is reflected in the SEM's 2D result.

Approach: Direct comparison by measuring an identical location of a line pattern by SEM and an atomic force microscopy (AFM) with the tip-tilting technique that is capable of measuring the 3D sidewall. The line pattern has vertical stripes on the sidewall due to its fabrication process. Measured line edge profiles were analyzed using power spectral density, height-height correlation function, and autocorrelation function.

Results: Line edge profiles measured by SEM and AFM were well matched except for noise level. Frequency and scaling analyses showed that SEM profile contained high noise and had lost a property of self-affine fractals in contrast to AFM.

Conclusions: In the case of the line pattern with vertical stripes on the sidewall, SEM profile is generally consistent with 3D sidewall shape. The AFM-based LER measurement technique is useful as LER reference metrology to evaluate other LER measurement techniques.

(C) The Authors. Published by SPIE under a Creative Commons Attribution 4.0 Unported License. Distribution or reproduction of this work in whole or in part requires full attribution of the original publication, including its DOI. [DOI: 10.1117/1.JMM.19.4.044001]
\end{abstract}

Keywords: line edge roughness; roughness parameters; power spectral density; height-height correlation function; autocorrelation function; reference metrology; scanning electron microscopy; atomic force microscopy.

Paper 20036 received Jul. 31, 2020; accepted for publication Oct. 13, 2020; published online Oct. 23, 2020.

\section{Introduction}

Line edge roughness (LER) measurement of a nanoscale line pattern, which is important for the evaluation of micro/nano fabrication techniques in the semiconductor industry, poses a metrology challenge. In the fabrication process, critical-dimension scanning electron microscopy (CD-SEM) is often used for measuring dimensions such as linewidth, contour, and LER. CD-SEM is often suitable for such measurements because of its relatively high spatial resolution and throughput. Contrarily, recent advanced lithography techniques involving more shrinkage and complicated 3D structures demand highly precise metrology techniques. Conventional SEM (including CD-SEM) is a top-view (2D) metrology technique, and is therefore not suitable for accurately measuring 3D structures. For LER measurements, although the 3D sidewall may involve unique line-edge profiles at each height, SEM measurement generates only one line-edge

*Address all correspondence to Ryosuke Kizu, r-kizu@aist.go.jp 
profile representing the 3D sidewall's roughness. Moreover, the SEM technique exhibits instrumental noise, which distorts measured line edge profiles and biases LER higher. Although CDSEM is necessary as an in-line metrology technique, such error sources can produce an unacceptable error in LER analysis. Therefore, quantitatively investigating the error is vital. ${ }^{1}$

LER is often evaluated using three times the standard deviation $(3 \sigma)$ of the distances between points forming a line-edge profile and its fitted linear line. To completely characterize a line-edge profile, the roughness exponent $(\alpha)$ and correlation length $(\xi)$ are required in addition to $\sigma$. These three parameters are often obtained from the power spectral density (PSD) or height-height correlation function (HHCF) of the line-edge profile., ${ }^{2,3}$ A correction method for LER analysis error caused by noise in SEM images was developed. ${ }^{4-6}$ The proposed method enables highly reproducible LER analysis, particularly for $\sigma$. However, if the true fine structure (roughness) of a line edge was lost owing to measurement noise and/or inadequate SEM spatial resolution, correctly reconstructing the roughness parameters and evaluating their accuracy is difficult, particularly with respect to $\alpha$. This is because $\alpha$ is determined by the PSD (or HHCF) shape in a highspatial-frequency (short length) region, where the PSD's (HHCF's) shape is distorted by noise and/or lack of resolution.

In general, the sidewall roughness of a 3D line pattern affects the device performance and production yields, ${ }^{7}$ which is caused by an imperfection of the lithography process. Ideally speaking, the required measurement is the 3D sidewall shape. However, considering industrial device inspection using CD-SEM, simplifying the 3D sidewall shape as a line-edge profile ( $x y$-plot) is preferable, for compatibility with the CD-SEM 2D results. Therefore, measuring the 3D sidewall necessitates high spatial resolution to obtain a high-resolution 2D line-edge profile in a certain way and calculate the roughness parameters. For example, it was proposed that the measured 3D sidewall image is divided into 100 line-edge profiles in the height direction (a horizontal slicing process), and then the line-edge profile at half height is considered a representative of the $3 \mathrm{D}$ sidewall shape along the line pattern. ${ }^{8}$

Hybrid metrology is the concept of combining two or more measurement techniques. For example, a measurement technique with high accuracy, referred to as reference metrology, ${ }^{9}$ is used to evaluate another measurement technique with lower accuracy (but with important characteristics such as high throughput). In LER metrology, reference metrologies using transmission electron microscopy (TEM) and atomic force microscopy (AFM) have been proposed. ${ }^{8,10-13}$ The TEM-based LER measurement technique is applicable to a real device sample with a complex structure, although it is destructive and exhibits low spatial resolution in the height direction. ${ }^{10,11}$ In contrast, AFM-based techniques enable the precise measurement of 3D sidewall shape ${ }^{8,12-19}$ The two principal AFM-based LER measurement techniques are CD-AFM that employs a flared tip and tilting-AFM that uses a tilted sharp tip for scanning the vertical sidewall. Although both techniques are nondestructive and enables 3D control of tip position with high spatial resolution, the AFM's tip cannot get into dense and complex patterns. CD-AFM was mainly developed for linewidth measurements; however, the large diameter of the flared tip (typically several tens of nanometers) causes relatively low spatial resolution along the line pattern. ${ }^{12,13,15,18}$ In contrast, tilting-AFM employs a sharp tip (typical diameter of several nanometers), so the spatial resolution along the line pattern is high. ${ }^{8,16}$ Therefore, tilting-AFM is more suitable as LER reference metrology than CD-AFM.

A direct comparison of the line-edge profiles measured by SEM and the reference metrology at an identical location is effective for investigating the relation between the two profiles. A lineedge profile produced by the lithography process contains nanometric structures that are difficult to be distinguished from SEM's noise. The nanometric structure should be measured by reference metrology and compared with SEM results at an identical location. Roughness parameters are suitable for comparing the line-edge profiles measured at an identical location. Several studies examined the shape of nanostructures at an identical location using SEM and AFM. Azarnouche et al. ${ }^{5}$ and Faurie et al., ${ }^{12}$ for example, measured the line width roughness of a photoresist pattern and silicon pattern to investigate the electron-beam (EB) impact on the patterns and accurately estimate the LER. However, only $3 \sigma$ values were compared in these studies, which is insufficient for comparison because it ignores $\alpha$ and $\xi$.

In this study, we compared the SEM and tilting-AFM measurements. The tilting-AFM, which is capable of measuring the 3D sidewall shape, acted as the reference metrology. An 
identical location of a Si line pattern was measured by SEM and the tilting-AFM. We evaluated the differences between the two line-edge profiles using PSD and HHCF. The reliability of the estimated roughness parameters was checked by comparing theoretical and experimental autocorrelation functions.

\section{Experimental Setup}

The measurement sample was a Si line pattern produced by EB lithography and dry etching (from NTT Advanced Technology Corp.) having a height and sidewall angle of $\sim 200 \mathrm{~nm}$ and $86^{\circ}$, respectively. The SEM and AFM instruments were a field-emission scanning electron microscope (S-4800, Hitachi High-Technologies, Corp.) and a metrological tilting-atomic force microscope (tilting-mAFM) developed by National Metrology Institute of Japan, ${ }^{20}$ respectively.

The measurement conditions for SEM were as follows: acceleration voltage of $5 \mathrm{kV}, 40,000 \times$ magnification, number of frames 32, and image size of $1920 \times 2560(2381 \mathrm{~nm} \times 3174 \mathrm{~nm}$, pixel size of $1.24 \mathrm{~nm} \times 1.24 \mathrm{~nm}$ ). Distinguishing the line-edge profile was not easy owing to noise; therefore, for averaging, SEM images were taken eight times at the same location. TiltingmAFM measurement was performed by the same way described in Ref. 8. Measurement conditions were as follows: tip-tiling-angle and inclining angle of servo axis were $14^{\circ}$ and $30^{\circ}$, respectively; image size (3D point cloud) was 2000 points $\times 500$ lines; and measurement area was $4000 \mathrm{~nm} \times 200 \mathrm{~nm}$. The fast scan direction was along the line pattern, and the 3D sidewall shape was scanned from the bottom to top surface through the sidewall [Fig. 1(a)]. SEM measurement was performed, followed by the tilting-mAFM measurements. The measured location was determined by an alignment mark (end edge along the line pattern), so that the field-of-views (FOVs) of the SEM and tilting-mAFM overlapped (note that the tilting-mAFM measures only one sidewall per measurement), as shown in Fig. 1(b).

\section{Results and Discussion}

\subsection{Line-Edge Profiles Measured by SEM and AFM at an Identical Position}

Figure 2(a) shows one of the eight SEM images rotated $90^{\circ}$ clockwise after eliminating the unnecessary portion. The top and bottom line edges in the image exhibit peak brightness. In this study, we considered the brightest pixel as the edge pixel (without interpolation process) to simplify the edge-detection algorithm and prevent serious detection errors. Figure 2(b) shows one of the eight line-edge profiles (orange) and the average of the eight line-edge profiles (red). The single line-edge profile is characterized by high noise that was quantized by a length of $1.24 \mathrm{~nm}$ owing to the pixel size of the SEM image. The averaged line-edge profile was obtained by considering the drift of the eight line-edge profiles ${ }^{5,21}$ and shifting to be well matched. Consequently, the averaged line-edge profile produces a clearer shape because of less noise and quantization.

Figure 3(a) shows a 3D sidewall image expressed as a 3D point cloud measured by the tiltingmAFM, while Fig. 3(b) shows an enlarged image of the sidewall portion with vertical stripes

(a)

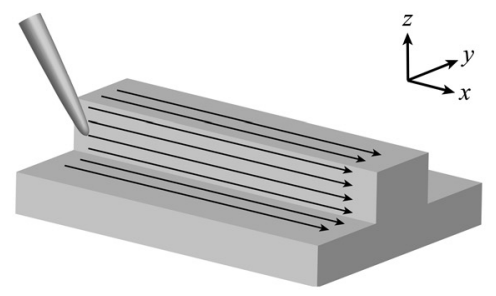

(b) $y$

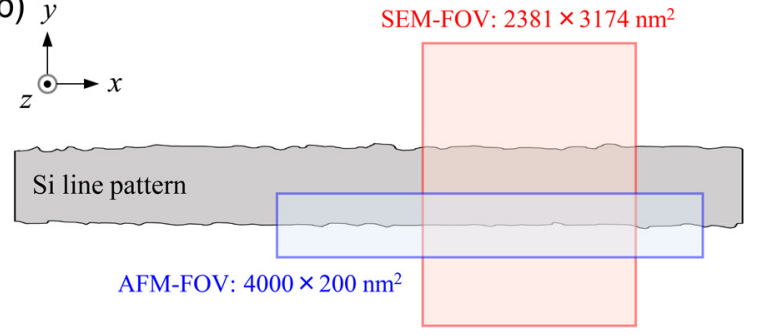

Fig. 1 (a) Illustration of the fast scan direction along the line pattern. The 3D sidewall shape is scanned from the bottom to top surface through the sidewall by the tilting-mAFM. (b) The FOVs of the SEM and tilting-mAFM overlapped for the direct comparison. 
Kizu et al.: Direct comparison of line edge roughness measurements by SEM and a metrological...

(a)
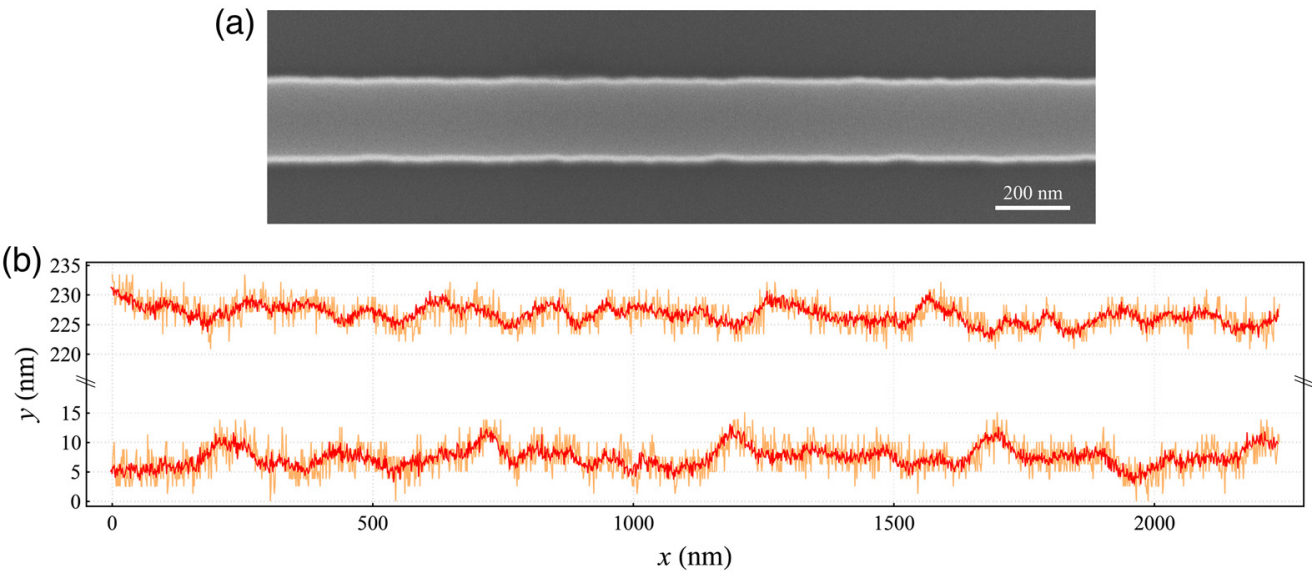

Fig. 2 (a) One of the eight SEM images of the Si line pattern. (b) One of the eight line-edge profiles (orange) and the averaged line-edge profile (red).
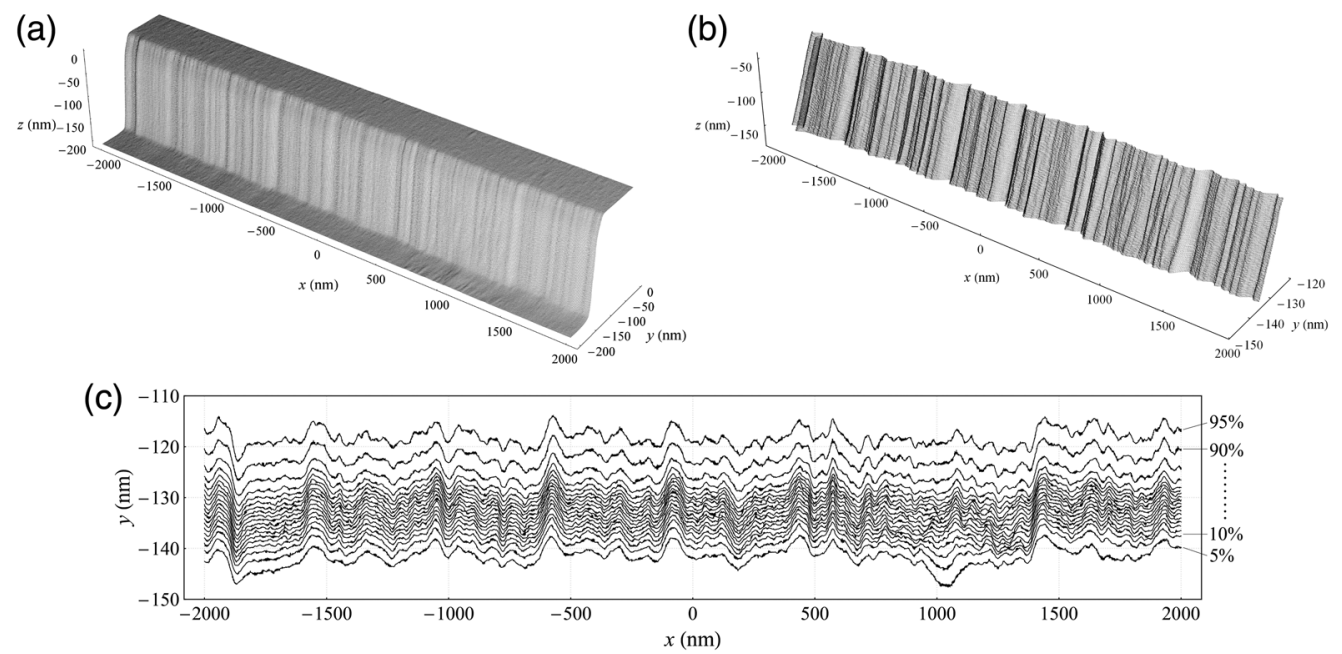

Fig. 3 (a) AFM image of a sidewall expressed as a 3D point cloud. The scale of each axis is modified for visibility. (b) An enlarged sidewall image of (a). (c) Line-edge profiles ( $x y$ plot) at every $5 \%$ height $(z)$ interval from $5 \%$ to $95 \%$.

clearly visible on the sidewall. We divided it into 200 line-edge profiles (with no filtering, averaging, and interpolation processes) at a 1-nm height interval from the bottom to top as an $x y$ plot (like a horizontally sliced profile) through the same data processing in the literature. ${ }^{8}$ In Fig. 3(c), the line-edge profiles at every 5\% height from 5\% to $95 \%$ are illustrated. This Si line pattern has a sidewall angle of $\sim 86^{\circ}$; therefore, the line-edge profile at each height has an offset in the $y$ direction. The line-edge profiles at 5\%,85\%, 90\%, and 95\% exhibit large offsets owing to the AFM's tip convolution. However, the convolution effect is almost uniform at the same measurement height; therefore, the distortion of the line-edge profile would be negligible.

Figure 4 shows SEM profiles of lower edge [one profile (orange) and the averaged (red)] in Fig. 2, and the tilting-mAFM profile at the 50\% height (blue). The horizontal (xy) scaling of the SEM profiles was adjusted to 1.016 times, to accommodate with that of the tilting-mAFM profile, which is traceable to the SI unit of length. If the scaling error of SEM remained, obtained $\sigma$ and $\xi$ also had errors whereas no effect on $\alpha$ was observed. The figure apparently indicates that the sidewall at an identical location of the sidewall was measured by the SEM and tilting-mAFM. For precise comparison, the tilting-mAFM profile was cut at the same $x$-range of the SEM profiles, which corresponds to the $x$-range from -1770 to $480 \mathrm{~nm}$ of Fig. 3(c). The LER $(3 \sigma)$ calculated for one of the eight SEM profiles, the averaged SEM profile, and the tilting-mAFM profile 
Kizu et al.: Direct comparison of line edge roughness measurements by SEM and a metrological...

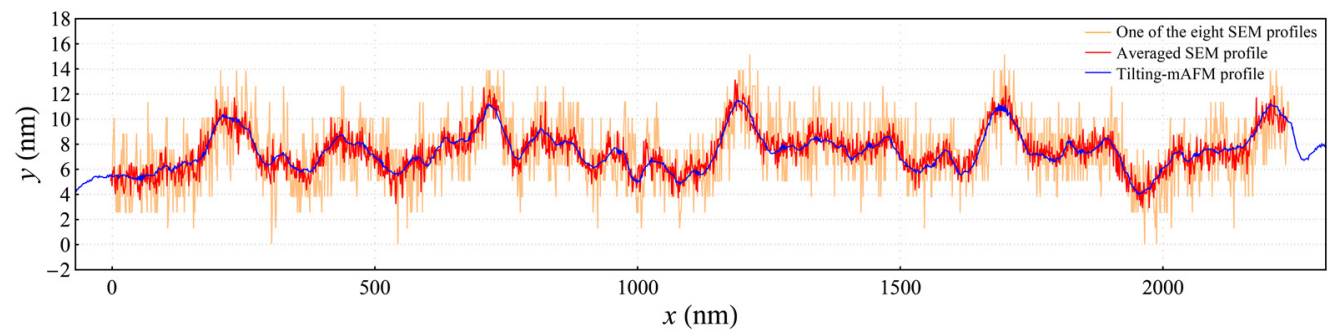

Fig. 4 Line-edge profiles for one of the eight SEM profiles (orange), the averaged SEM profile (red), and the tilting-mAFM profile (blue).

Table 1 LERs $(3 \sigma)$ calculated for one of the eight SEM profiles, the averaged SEM profile, and the tilting-mAFM profile.

\begin{tabular}{lc}
\hline \hline Line-edge profiles & $3 \sigma(\mathrm{nm})$ \\
\hline One of the eight SEM profiles & 7.69 \\
Averaged SEM profile & 4.94 \\
Tilting-mAFM profile & 4.53 \\
\hline \hline
\end{tabular}
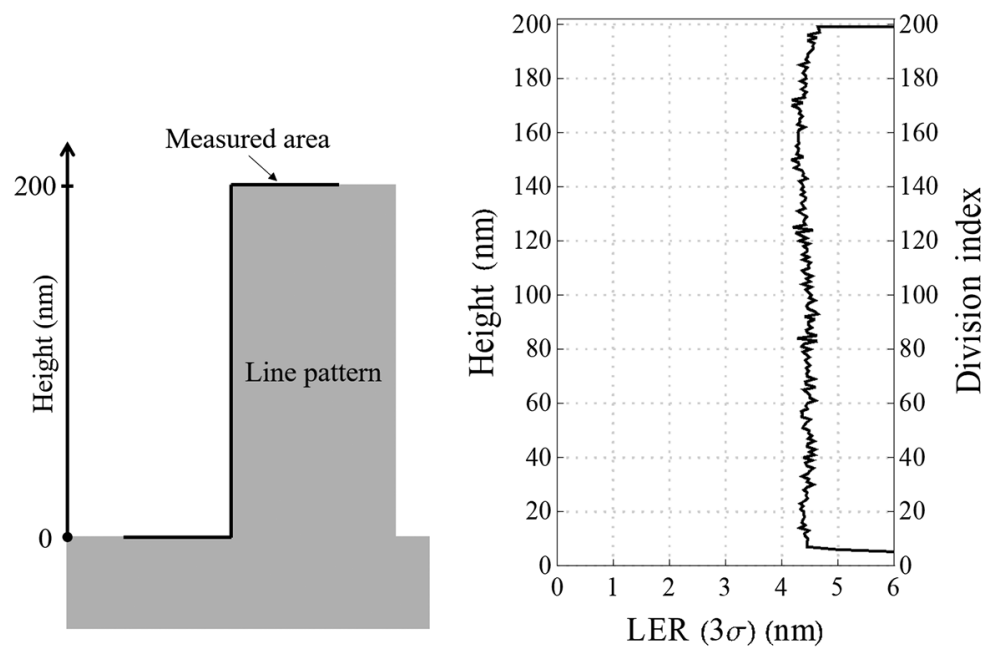

Fig. 5 LER $(3 \sigma)$ distribution along height.

are given in Table 1. Considering the LER of the tilting-mAFM profile as a reference value, LERs for one of the eight SEM profiles and the averaged SEM profile are larger by 2.75 and $0.41 \mathrm{~nm}$, respectively. It is worthy to note that LER $(3 \sigma)$ distribution along the height showed low height dependency because the sidewall of the line pattern exhibited vertical stripes, as shown in Fig. 5.

\subsection{PSD, HHCF, and Autocorrelation Function Analysis}

PSD and HHCF are often used for frequency and scaling analyses of a line-edge profile. ${ }^{2,3}$ PSD is calculated using discrete Fourier transform, while HHCF $[G(r=m d)(m=1,2, \ldots, N-1$, $N$ is the total number of points in a profile)] is calculated as

$$
G(r=m d)=\left[\frac{1}{N-m} \sum_{n=1}^{N-m}\left(x_{n+m}-\overline{x_{n}}\right)^{2}\right]^{1 / 2}
$$


where $x_{n}(n=0,1,2, \ldots, N-1)$ are resampled points with a constant interval $d$. The interval of points in a profile of tilting-mAFM is not uniform. The points were resampled at 2-nm intervals by interpolation to enable the calculation of the PSD and HHCF of the line-edge profile. The SEM profile was handled similarly to match the conditions for the tilting-mAFM.

Figure 6 shows the average of eight PSDs from eight SEM profiles (orange), the PSD of the averaged SEM profile (red), and the PSD of the tilting-mAFM profile at half height (blue). A rectangular window was used as the window function for the PSD calculations. The average of eight PSDs from eight SEM profiles has the flat noise floor in the high-frequency region. The PSD of the averaged SEM profile is not the average PSD, causing a variation instead of the flat noise floor in the high-frequency region. The averaged SEM profile exhibits lower noise than one of the eight SEM profiles; therefore, it produces a lower PSD amplitude at high frequencies. The PSD of the tilting-mAFM profile is lower than the noise floor of the SEM profiles in the high-frequency region. The wide dynamic range of the PSD of the tilting-mAFM profile and its power law behavior in the high-frequency region indicate lower noise and enough resolution. The PSDs of the averaged SEM profile and the tilting-mAFM profile show similar shapes at frequencies below $0.022 \mathrm{~nm}^{-1}$. Considering the AFM data as reference data, we can determine that SEM profiles are distorted by noise and/or lack of resolution at frequencies higher than $0.022 \mathrm{~nm}^{-1}$. It is worthy to note that although there are edge-detection algorithms which would enable lower noise for SEM profile, ${ }^{22}$ the improvement of the noise level by the advanced algorithms is less than superiority of the AFM and do not affect the discussion in this study.

Figure 7 shows (i) the average of eight HHCFs for eight SEM profiles (orange), (ii) the HHCF of the averaged SEM profile (red), and (iii) the HHCF of the tilting-mAFM profile (blue).

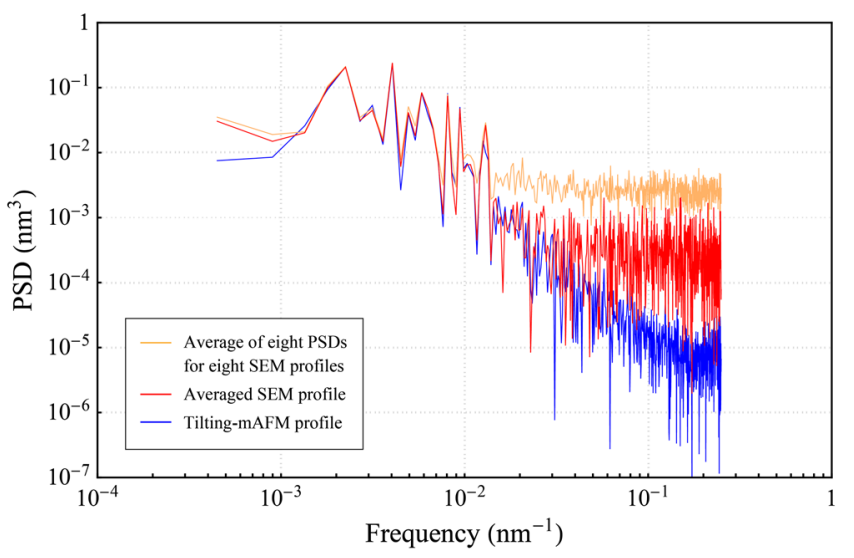

Fig. 6 Average of eight PSDs for eight SEM profiles (orange), PSD of the averaged SEM profile (red), and PSD of the tilting-mAFM profile (blue).

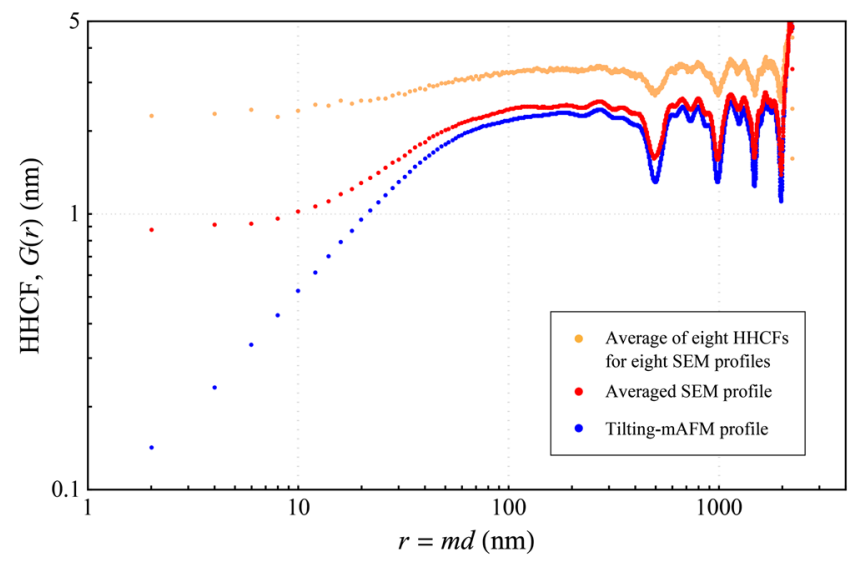

Fig. 7 Average of eight HHCFs for eight SEM profiles (orange), the HHCF of the averaged SEM profile (red), and the HHCF of the tilting-mAFM profile (blue). 
Kizu et al.: Direct comparison of line edge roughness measurements by SEM and a metrological...

Table 2 Roughness parameters calculated from the HHCFs of each line-edge profile.

\begin{tabular}{lccc}
\hline \hline Line-edge profiles & $\sigma(\mathrm{nm})$ & $\alpha$ & $\xi(\mathrm{nm})$ \\
\hline One of eight SEM profiles & 2.37 & - & 23 \\
Averaged SEM profile & 1.75 & - & 48.8 \\
Tilting-mAFM profile & 1.64 & 0.87 & 42.6 \\
\hline \hline
\end{tabular}

HHCF-(i) completely differs from HHCF-(ii) and HHCF-(iii) at all distances. HHCF-(i) and HHCF-(ii) exhibit a flat region in the smaller- $r$ region owing to the noise. HHCF-(iii) linearly increases (indicating power law behavior) from 2 to $30 \mathrm{~nm}$ and becomes saturated and oscillates beyond $30 \mathrm{~nm}$. HHCF-(ii) is similar to HHCF-(iii) only in the larger- $r$ region. This suggests that SEM failed to accurately measure the short-distance characteristics. Table 2 shows the roughness parameters $\sigma, \alpha$, and $\xi$ calculated from these three HHCFs by manually drawing the power law lines and their saturation lines. ${ }^{2,3}$ This method enables us to estimate roughness parameters easily and approximately. It is worthy to note that SEM's HHCFs showed flat region in smaller- $r$ region, and it was difficult to apply the power-law fitting, which requires self-affine property of the profile. A different method such as an unbiased correction method ${ }^{6}$ would be required to evaluate $\alpha$ from the SEM's HHCFs. Considering HHCF-(iii) as the reference, the roughness parameters for HHCF-(ii) show differences of 0.11 and $6.2 \mathrm{~nm}$ for $\sigma$ and $\xi$, respectively. $\sigma$ and $\xi$ yield relatively small errors because of the saturation line similarity between HHCF-(ii) and HHCF-(iii). On the other hand, SEM's $\alpha$ could not be obtained owing to the distortion of HHCF(ii) in the smaller- $r$ region.

$\sigma$ and $\xi$ are determined using the saturation value primarily from the long-distance component of the HHCF; therefore, these are relatively stable against noise. Contrarily, $\alpha$ is determined from the decreasing pattern of the HHCF from the long- to short-distance region. The distortion of the HHCF in the short-distance region makes it difficult to obtain $\alpha$ because the distortion implies the analyzed profile did not have the self-affine property. Figures 6 and 7 show that the PSD and HHCF of tilting-mAFM show less noise and enough spatial resolution. In particular, the HHCF of tilting-mAFM shows very low noise, such that it enables stable calculation of the roughness parameters without any correction method. These results indicate that the use of tilting-mAFM as a reference metrology is suitable for evaluating the roughness parameters calculated from SEM measurement.

Figure 8 shows the autocorrelation functions $\operatorname{ACF}(r=m d)(m=0,1,2, \ldots, N-1, N$ is the total number of profiles) of the averaged SEM profile (red dots) and the tilting-mAFM profile (blue dots), which are calculated as follows:

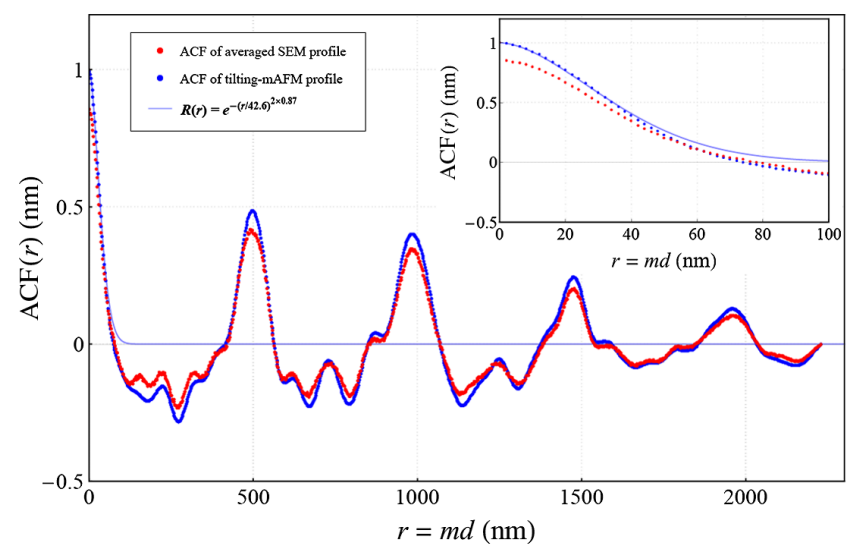

Fig. 8 Plot of the calculated ACFs for the averaged SEM profile (red dots) and the tilting-mAFM profile (blue dots), and the model autocorrelation function for the tilting-mAFM profile (light blue line). The inset is an enlarged plot of part of the $x$ axis. 


$$
A C F(r=m d)=\frac{1}{N-m} \sum_{n=1}^{N-m}\left(x_{n}-\overline{x_{n}}\right)\left(x_{n+m}-\overline{x_{n}}\right) / \sum_{n=1}^{N-m}\left(x_{n}-\overline{x_{n}}\right)^{2}
$$

The two autocorrelation functions fluctuate significantly and display similarity at large $r$ (lag distance) values. A model autocorrelation function, which is reasonable for self-affine fractal profiles $^{3,23}$ is expressed as follows:

$$
R(r)=\sigma^{2} e^{-(r / \xi)^{2 \alpha}}
$$

The blue solid line in Fig. 8 was drawn using Eq. (3), with the parameters of $\sigma=1$ [corresponding to $A C F(r)$ normalized by the variance of $x_{n}$ ] and the $\alpha$ and $\xi$ of tiltingmAFM profile from HHCF (Table 2). For the averaged SEM profile, a solid line [Eq. (3)] with parameters from HHCF (Table 2) could not be written due to the absence of $\alpha$. The relationship between HHCF $[G(r)]$ and $\mathrm{ACF}(r)$ is as follows: ${ }^{2,3}$

$$
G^{2}(r)=2 \sigma^{2}[1-A C F(r)] .
$$

$A C F(r)$ is ideally the same as $R(r)$. Thus, if the roughness parameters obtained from HHCF are correct, the $R(r)$ calculated from them matches with the $A C F(r)$. The inset in Fig. 8 shows the blue dots and light blue line agree in shape for small $r$, particularly $r<40 \mathrm{~nm}$. This indicates the consistency between the two analyses by HHCF and the autocorrelation function for the tilting-mAFM profile.

\subsection{Discussion on LER Reference Metrology}

In this study, the sidewall of the line pattern exhibits vertical stripes and low height dependency. Therefore, the SEM profile was generally consistent with the tilting-mAFM profile at half-height extracted from the 3D AFM image. However, if a line pattern exhibiting height dependency, e.g., the gate-all-around FET structure, is measured, the line-edge profiles of the SEM and tiltingmAFM would differ significantly. The results of this study imply that a line pattern with vertical stripes on the sidewall is suitable for evaluating the SEM's performance for LER measurement. Therefore, we propose the use of an LER reference standard, i.e., a line pattern with the sidewall with vertical stripes. The 3D sidewall shape was preliminarily measured by the reference metrology (e.g., tilting-mAFM), and the line-edge profiles at every height and associated roughness parameters were calibrated; then, the line pattern was utilized for evaluating SEM instruments. The LER reference standards should be regularly measured by SEM to assess its performance for LER measurement. That is a similar way of scale calibration procedure of the CD-SEM, wherein preliminary calibrated 1D grating (pitch) standards are incorporated into the CD-SEM instruments. Fortunately, such line patterns (having vertical stripes) are easily fabricated by conventional lithography techniques. In contrast, several important factors pertaining to reference standards (such as sample deterioration against the EB of the SEM and SEM-image dependency on the line pattern's height, sidewall angle, and SEM's accelerating voltage) must be investigated in detail for the practical use of the LER reference standards. The reference metrology for measuring the 3D sidewall's shape can be used for evaluating SEM and other dimensional-measurement techniques such as tilt-beam (or 3D-) SEM and normal AFM, and will be useful for providing reference input data for deep learning-aided LER metrology. ${ }^{24,25}$

\section{Conclusion}

Conventional LER measurement by SEM faces difficulty in precisely measuring the roughness parameters owing to noise. Even with a correction method having high reproducibility, the evaluation of the accuracy remains challenging. Moreover, although the actual line pattern exhibits a 3D sidewall, the line-edge profile measured by SEM involves the top-view (2D) measurement; hence, there is no way to verify the constituents of the 3D sidewall reflected in the SEM result. In this study, we used tilting-mAFM, which is capable of measuring a $3 \mathrm{D}$ sidewall with 
SI-traceability as the reference metrology, and directly compared the SEM and tilting-mAFM for identical locations, for precise evaluation of the SEM's performance for LER measurement. The tilting-mAFM data were processed to produce line-edge profiles for every 1-nm height from the bottom to top as an $x y$ plot. A line-edge profile at half height was analyzed using PSD, HHCF, and autocorrelation function. Results demonstrated that the tilting-mAFM involves low noise and capability of obtaining the roughness parameter without any correction method. The reference metrology for measuring a 3D sidewall shape by the tilting-mAFM can be used for the evaluation of SEM and other dimensional-measurement techniques. Future work will focus on comparing tilting-mAFM with the unbiased correction method $^{4-6}$ and investigating the error caused by tip-dilation effect that may make the roughness profile smooth.

\section{Acknowledgments}

This work was partially supported by JSPS KAKENHI (Grant No. JP19K14865) and conducted at the Advanced Industrial Science and Technology Nano-Processing Facility, supported by "Nanotechnology Platform Program" of the Ministry of Education, Culture, Sports, Science and Technology, Japan. Part of this work was presented at the 2020 SPIE Advanced Lithography Conference. $^{26}$

\section{References}

1. G. F. Lorusso et al., "Need for LWR metrology standardization: the IMEC roughness protocol," J. Micro/Nanolithogr. MEMS MOEMS 17, 041009 (2018).

2. V. Constantoudis et al., "Quantification of line-edge roughness of photoresists. II. Scaling and fractal analysis and the best roughness descriptors," J. Vac. Sci. Technol. B 21, 10191026 (2003).

3. V. Constantoudis et al., "Line edge roughness and critical dimension variation: fractal characterization and comparison using model functions," J. Vac. Sci. Technol. B 22, 1974-1981 (2004).

4. J. S. Villarrubia and B. D. Bunday, "Unbiased estimation of linewidth roughness," Proc. SPIE 5752, 480-488 (2005).

5. L. Azarnouche et al., "Unbiased line width roughness measurements with critical dimension scanning electron microscopy and critical dimension atomic force microscopy," J. Appl. Phys. 111, 084318 (2012).

6. G. F. Lorusso et al., "Unbiased roughness measurements: subtracting out SEM effects," Microelectron. Eng. 190, 33-37 (2018).

7. S. Oh and C. Shin, "3-D quasi-atomistic model for line edge roughness in nonplanar MOSFETs," IEEE Trans. Electron Devices 63, 4617 (2016).

8. R. Kizu et al., "Line edge roughness measurement on vertical sidewall for reference metrology using a metrological tilting atomic force microscope," J. Micro/Nanolithogr. MEMS MOEMS 19, 014003 (2020).

9. V. Ukraintsev and B. Banke, "Review of reference metrology for nanotechnology: significance, challenges, and solutions," J. Micro/Nanolithogr. MEMS MOEMS 11, 011010 (2012).

10. K. Takamasu et al., "3D-profile measurement of advanced semiconductor features by using FIB as reference metrology," Proc. SPIE 10145, 101451A (2017).

11. K. Takamasu et al., "Linewidth roughness of advanced semiconductor features using focused ion beam and planar-transmission electron microscope as reference metrology," J. Micro/Nanolithogr. MEMS MOEMS 17, 041010 (2018).

12. P. Faurie, J. Foucher, and A.-L. Foucher, "The LER/LWR metrology challenge for advance process control through 3D-AFM and CD-SEM," Proc. SPIE 7520, 75200F (2009).

13. M. Fouchier, E. Pargon, and B. Bardet, "An atomic force microscopy-based method for line edge roughness measurement," J. Appl. Phys. 113, 104903 (2013).

14. C. Nelson et al., "Comparison of metrology methods for quantifying the line edge roughness of patterned features," J. Vac. Sci. Technol. B 17, 2488-2498 (1999). 
15. N. G. Orji et al., "Line edge roughness metrology using atomic force microscopes," Meas. Sci. Technol. 16, 2147-2154 (2005).

16. S.-J. Cho et al., "Three-dimensional imaging of undercut and sidewall structures by atomic force microscopy," Rev. Sci. Instrum. 82, 023707 (2011).

17. O. Fursenko et al., "Characterization of Si nanowaveguide line edge roughness and its effect on light transmission," Mater. Sci. Eng. B 177, 750-755 (2012).

18. G. Dai et al., "Measurements of CD and sidewall profile of EUV photomask structures using CD-AFM and tilting-AFM," Meas. Sci. Technol. 25, 044002 (2014).

19. D. Hussain et al., "Advances in the atomic force microscopy for critical dimension metrology," Meas. Sci. Technol. 28, 012001 (2017).

20. R. Kizu et al., "Development of a metrological atomic force microscope with a tip-tilting mechanism for 3D nanometrology," Meas. Sci. Technol. 29, 075005 (2018).

21. S.-B. Wang et al., "Practical and bias-free LWR measurement by CDSEM," Proc. SPIE 6922, 692222 (2008).

22. C. Mack, "Comparing edge detection algorithms: their impact on unbiased roughness measurement precision and accuracy," Proc. SPIE 11325, 113250P (2020).

23. G. Palasantzas et al., "Roughness spectrum and surface width of self-affine fractal surfaces via the K-correlation model," Phys. Rev. B 48, 14472 (1993).

24. N. Chaudhary et al., "Line roughness estimation and Poisson denoising in scanning electron microscope images using deep learning," J. Micro/Nanolithogr. MEMS MOEMS 18, 024001 (2019).

25. E. Giannatou et al., "Deep learning denoising of SEM images towards noise-reduced LER measurements" Microelectron. Eng. 216, 111051 (2019).

26. R. Kizu et al., "Comparison of SEM and AFM performances for LER reference metrology," Proc. SPIE 11325, 113250Q (2020).

Biographies of the authors are not available. 